\section{Clostridium difficile Infection in Hospitalized Patients with Cystic Fibrosis}

Patients with cystic fibrosis (CF) may be at a higher risk for developing Clostridium difficile infection (CDI) due to a greater exposure to hospitals and antibiotics. ${ }^{1}$ A propensity score-matched case-control study was performed using the Healthcare Cost and Utilization Project Nationwide Inpatient Sample, 2002-2010. We included patients 18-44 years of age; the cutoff was chosen to avoid skewed data. ${ }^{2}$ We extracted entries with a discharge diagnosis of CF (International Classification of Diseases, Ninth Revision, Clinical Modification [ICD9-CM] codes 277.00-277.09), CDI (ICD-9-CM code 008.45), and CF with lung transplantation (V42.6, 996.84, 33.5, 33.50, 33.51, and 33.52); demographic details; and comorbid conditions to calculate the Charlson comorbidity index (Deyo modification). ${ }^{3,4}$ Outcome variables included mortality, total charges, length of stay (LOS), and colectomy (ICD-9-CM codes $45.7 \mathrm{x}, 45.8)$. Case patients were categorized into those with (1) a discharge diagnosis of CDI but no diagnosis of CF (CDI only) and (2) a discharge diagnosis of both CF and CDI (CF plus CDI). Statistical analyses were performed using SAS version 9.3. Cohort matching was performed as described elsewhere with propensity scores and a greedy matching algorithm in a $1: 5$ matching ratio ( $\mathrm{CF}$ and without $\mathrm{CF}){ }^{5}$ The $\chi^{2}$ test, Mann-Whitney $U$ test, and Cochrane Armitage test for trend and regression modeling were applied appropriately.

CDI incidence among hospitalized patients 18-44 years of age with CF increased $0.9 \%$ to $1.6 \%$ from 2002 to 2010 . Concurrently, the disease incidence in the age-matched hospitalized non-CF population increased $0.2 \%$ to $0.3 \%$. In both groups, this represented a significant increased trend $(P<$ $.05)$. In 2010 , there were a total of $9,706,097$ hospital discharges among patients 18-44 years of age. There were 32,541 discharges with CDI, 19,278 with CF, and 302 with both CF and CDI. Patients with CF had a higher risk for CDI after matching for demographic characteristics and comorbidities (odds ratio [95\% confidence interval (CI)], 3.0 [2.6-3.5]) and in patients with CF and lung transplantation $(1,849$ hospital discharges), the incidence was even higher $(3.1 \%$ vs $1.4 \% ; P<.05)$. Using a multiple variable regression model to control for demographic factors and comorbidities, the CF plus CDI group demonstrated greater risks of death (adjusted odds ratio [95\% CI], 3.1 [1.9-5.1]) and colectomy (adjusted odds ratio [95\% CI], $2.6[1.3-5.3])$, and higher hospital charges (adjusted regression coefficient [95\% CI], \$42,000 [\$22,000-\$62,000]; Table 1). The difference in LOS did not тав в 1. Outcomes Associated with Clostridium difficile Infection in Hospitalized Patients with Cystic Fibrosis Age 18-44 Years Using a Multiple Variable Regression Model

\begin{tabular}{lcc}
\hline Outcome & $\begin{array}{c}\text { Adjusted odds } \\
\text { ratio }(95 \% \mathrm{CI})\end{array}$ & $\begin{array}{c}\text { Adjusted regression } \\
\text { coefficient }(95 \% \mathrm{CI})\end{array}$ \\
\hline Mortality & $3.1(1.9-5.1)$ & $\ldots$ \\
Colectomy & $2.6(1.3-5.3)$ & $\ldots$ \\
Length of stay, days & $\ldots$ & $3.3(0.81-5.8)$ \\
Hospital charges, $\$$ & $\ldots$ & $42,000(22,000-62,000)$ \\
\hline
\end{tabular}

Note. Data adapted from the Healthcare Cost and Utilization Project Nationwide Inpatient Sample sponsored by the Agency for Healthcare Research and Quality, 2010. Outcomes adjusted for age, sex, race, insurance status, geographic location of care, median household income quartile by zip code, hospital setting and hospital teaching status, and comorbid conditions. CI, confidence interval.

achieve statistical significance (adjusted regression coefficient [95\% CI], 3.3 days [0.81-5.8 days]).

This study represents, to our knowledge, the first use of multi-institutional data to investigate CDI in patients with CF. Contrary to earlier reports, ${ }^{1,6}$ patients with CF $18-44$ years of age demonstrated a threefold greater risk for developing CDI than age-matched control subjects; individuals with CF and lung transplantation were especially vulnerable. In our study population of hospitalized patients with CF, CDI was associated with overall worse outcomes and an increased incident trend of disease. Patients with CF have several risk factors for CDI, including increased frequency of hospitalization and use of antibiotics, decreased gastric acid, and impaired immunity, ${ }^{7}$ which may be even more evident in the subgroup who have undergone lung transplantation. ${ }^{8}$ Study limitations include using ICD-9-CM codes to identify cases and an absence of pharmacy and laboratory data and information regarding previous hospitalizations. Interpreting a $C$. difficile-positive test result for patients with $\mathrm{CF}$ is difficult given a high rate of asymptomatic carriage in this population. ${ }^{1,6}$ However, it is unusual to perform CDI testing in the absence of diarrhea, and we believe our patients represented true infection and not colonization. We conclude that CDI is a significant complicating factor in hospitalized adults with $\mathrm{CF}$ who are at risk of more severe disease; early diagnosis and aggressive treatment is imperative. Future studies should define the association of CDI and antibiotic use in this population.

\section{ACKNOWLEDGMENTS}

We acknowledge Healthcare Cost and Utilization Project (HCUP) Nationwide Inpatient Sample sponsored by the Agency for Healthcare Research and Quality that contribute to HCUP (http://www.hcup-us.ahrq .gov/db/hcupdatapartners.jsp). 
Potential conflicts of interest. All authors report no conflicts of interest relevant to this article. All authors submitted the ICMJE Form for Disclosure of Potential Conflicts of Interest, and the conflicts that the editors consider relevant to this article are disclosed here.

Chaitanya Pant, MD; ${ }^{1}$ Thomas J. Sferra, MD; ${ }^{2}$ Abhishek Deshpande, $\mathrm{MD}, \mathrm{PhD} ;^{3}$ Mojtaba Olyaee, $\mathrm{MD} ;^{1}$ Richard Gilroy, $\mathrm{MD} ;^{1}$ Michael P. Anderson, $\mathrm{PhD} ;^{4}$ Curtis Donskey, $\mathrm{MD}^{5}$

Affiliations: 1. Division of Gastroenterology, Hepatology and Nutrition, Department of Internal Medicine, University of Kansas Medical Center, Kansas City, Kansas; 2. Division of Pediatric Gastroenterology, Hepatology and Nutrition, Department of Pediatrics, Case Western Reserve University School of Medicine, University Hospitals Rainbow Babies and Children's Hospital, Cleveland, Ohio; 3. Medicine Institute Center for Value Based Care Research, Cleveland Clinic, Cleveland, Ohio; and Department of Infectious Diseases, Medicine Institute, Cleveland Clinic, Cleveland, Ohio; 4. Department of Biostatistics and Epidemiology, University of Oklahoma Health Sciences Center, Oklahoma City, Oklahoma; 5. Division of Infectious Diseases, Department of Medicine, Case Western Reserve University School of Medicine, Cleveland, $\mathrm{OH}$; and Geriatric Research, Education, and Clinical Center, Cleveland Veterans Affairs Medical Center, Cleveland, Ohio.

Address correspondence to Chaitanya Pant, MD, Division of Gastroenterology, Hepatology and Nutrition, Department of Internal Medicine, University of Kansas Medical Center, Kansas City, KS 66160 (pant55@yahoo .com).

Infect Control Hosp Epidemiol 2014;35(12):1547-1548

(C) 2014 by The Society for Healthcare Epidemiology of America. All rights reserved. 0899-823X/2014/3512-0017\$15.00. DOI: 10.1086/678607

\section{REFERENCES}

1. Bauer MP, Farid A, Bakker M, Hoek RA, Kuijper EJ, van Dissel JT. Patients with cystic fibrosis have a high carriage rate of nontoxigenic Clostridium difficile. Clin Microbiol Infect 2014;20(7): O446-O449.

2. Kopp BT, Wang W, Chisolm DJ, Kelleher KJ, McCoy KS. Inpatient healthcare trends among adult cystic fibrosis patients in the U.S. Pediatr Pulmonol 2012;47(3):245-251.

3. Deyo RA, Cherkin DC, Ciol MA. Adapting a clinical comorbidity index for use with ICD-9-CM administrative databases. J Clin Epidemiol 1992;45(6):613-619.

4. Quan H, Sundararajan V, Halfon P, et al. Coding algorithms for defining comorbidities in ICD-9-CM and ICD-10 administrative data. Med Care 2005;43(11):1130-1139.

5. Pant C, Deshpande A, Olyaee M, et al. Epidemiology of acute pancreatitis in hospitalized children in the United States from 2000-2009. PLOS ONE 2014;9(5):e95552.

6. Peach SL, Borriello SP, Gaya H, Barclay FE, Welch AR. Asymptomatic carriage of Clostridium difficile in patients with cystic fibrosis. J Clin Pathol 1986;39(9):1013-1018.

7. Egressy K, Jansen M, Meyer KC. Recurrent Clostridium difficile colitis in cystic fibrosis: an emerging problem. J Cyst Fibros 2013; 12(1):92-96.

8. Pant C, Anderson MP, O'Connor JA, Marshall CM, Deshpande A, Sferra TJ. Association of Clostridium difficile infection with outcomes of hospitalized solid organ transplant recipients: results from the 2009 Nationwide Inpatient Sample database. Transpl Infect Dis 2012;14(5):540-547. 